\title{
Estado nutricional, consumo de alimentos y actividad física en escolares mujeres de diferente nivel socioeconómico de Santiago de Chile
}

\author{
Sonia 0 livares $C^{1 a}$, Nelly Bustos $Z^{1 a}$, Lydia Lera $M^{1 b}$, \\ María Eugenia Zelada2c. \\ Nutritional status, food consumption \\ and physical activity in female school \\ children of different socioeconomic \\ levels from Santiago, Chile
}

Background: A high prevalence of obesity is the main public health problem in Chilean school children. Aim: To compare the nutritional status, consumption of selected foods and extracurricular physical activity (PA) habits in school children of different socioeconomic levels as a baseline for developing effective educational interventions. Material and methods: Crosssectional study that determined the body mass index, food consumption and physical activity with previously validated instruments in 202 and 358 girls from $3^{\text {rd }}$ to $8^{\text {th }}$ grade in schools of mediumhigh and low socioeconomic level (SEL) from Santiago, Chile, respectively. Results: Compared to their counterparts of low socioeconomic level (SEL), the prevalence of obesity was significantly lower in 8-9 year-old girls of medium high SEL (19\% and 9\%, respectively, $\mathrm{p}=0.012)$ and $12-13$ year-old $(12 \%$ and $2.5 \%$ respectively, $\mathrm{p}=0.008$ ). Also median daily intake of dairy products was higher in girls of medium high SEL ( 250 and $470 \mathrm{ml} /$ day, respectively). The intake of fruits and vegetables was similar ( $200 \mathrm{~g} / \mathrm{d}$ ); and the intake of bread was lower ( 230 and $70 \mathrm{~g} /$ day, respectively, $\mathrm{p} \varangle 0.01$ ). Consumption of energy-dense foods was lower in 10-13 year-old girls of medium high SEL (80 and $50 \mathrm{~g} /$ day, respectively, $\mathrm{p} \varangle 0.01$ ). $45 \%$ of 8-9 year-old girls and $35 \%$ of $12-13$ year-old girls of both SEL engaged in PA four or more times per week (NS). Conclusions: Although the prevalence of obesity in girls of medium-high SEL was not as high as in those from low SEL, it is still high. There is a need for educational interventions to improve their food and PA habits and to promote an environment that enhances healthy behaviors (Rev Méd Chile 2007; 135: 71-8).

(Key words: Eating; Life style; Obesity; Schools, Child Nutrition)

\footnotetext{
Recibido el 18 de octubre, 2005. Aceptado el 12 de octubre, 2006.

${ }^{1}$ Instituto de Nutrición y Tecnología de los Alimentos (INTA), Universidad de Chile. ${ }^{2}$ Colegio Saint John's Villa Academy, Santiago de Chile.

aNutricionistas, Magister en Ciencias de la Nutrición.

${ }^{b}$ Estadística. Doctora en Ciencias Matemáticas.

cProfesora de Educación Física.
}

Correspondencia a: Sonia Olivares. El Líbano 5524, Macul,

Santiago. Fax: 2214030. E mail: solivare@inta.cl 
L a obesidad infantil es considerada actualmente _una epidemia global y una crisis para la salud pública, tanto en países desarrollados como en desarrollo ${ }^{1,2}$, por su asociación con los factores de riesgo de enfermedades cardiovasculares ${ }^{3,4}$ y problemas en las articulaciones, desórdenes del sueño, cálculos a la vesícula y resistencia a la insulina, entre otros ${ }^{5}$.

Aunque en la etiología de la obesidad se reconoce la existencia de factores genéticos, metabólicos y ambientales, existe consenso en que las personas que consumen más calorías de las que gastan, acumulan depósitos de grasa ${ }^{1,2,6,7}$. Entre los factores que han contribuido a disminuir el gasto y aumentar la ingesta, se ha señalado la disminución de la actividad física, el tiempo que los niños destinan a ver televisión ${ }^{8-10}$, la publicidad de alimentos de alta densidad energética ${ }^{11} \mathrm{y}$ el mayor tamaño de las porciones que han conducido a un mayor consumo de energía ${ }^{12}$.

En Chile, la prevalencia de sobrepeso y obesidad infantil muestra una tendencia creciente y un rápido aumento en los últimos años ${ }^{13}$. Entre los años 2001 y 2005, se han realizado diversos estudios que determinaron el estado nutricional, el consumo de alimentos y la frecuencia con la que realizaban actividad física escolares urbanos y rurales asistentes a escuelas públicas en seis regiones del país, los que han formado parte de la línea base de intervenciones de educación en nutrición y promoción de la salud ${ }^{14-17}$. En estos estudios se encontraron prevalencias de sobrepeso cercanas a $20 \%$ y de obesidad entre $13 \%$ y $20 \%$ en niñas entre 8 y 13 años, sin diferencias significativas por región o sector urbano o rural ${ }^{18}$. Un estudio realizado en Santiago el año 2004, tampoco encontró diferencias significativas entre escolares de nivel socioeconómico (NSE) medio y bajo ${ }^{19}$.

En la última década, distintas investigaciones han coincidido en observar una insuficiente ingesta de lácteos, verduras y frutas, y un elevado consumo de alimentos de alta densidad energética y de bebidas con azúcar en todos los grupos de escola- res estudiados ${ }^{14,17,21}$. En niños de NSE medio, se observó una ingesta significativamente mayor de lácteos y menor de pan que en los de NSE bajo ${ }^{19}$.

Los estudios nacionales concuerdan en destacar la falta de actividad física y el considerable número de horas frente al televisor en los escolares de NSE bajo, así como su preferencia por los alimentos de alta densidad energética y bebidas publicitados en la televisión ${ }^{14,22,23}$.

El objetivo del presente estudio fue comparar el estado nutricional, el consumo de alimentos seleccionados y los hábitos de actividad física en escolares mujeres de NSE medio alto (información no disponible en el país), con los de escolares del mismo sexo pertenecientes al NSE bajo e identificar elementos que permitan diseñar estrategias educativas y de promoción en alimentación, nutrición y actividad física que resulten efectivas al ser aplicadas en los distintos grupos de escolares del país.

\section{MATERIAL Y MÉTODO}

Se trata de un estudio de corte transversal, en el que se determinó el estado nutricional, el consumo de algunos alimentos seleccionados y la actividad física en escolares mujeres de distinto NSE de la Región Metropolitana.

La muestra estuvo constituida por 204 niñas de $3^{\circ}$ a $8^{\circ}$ año básico de un colegio privado de NSE medio alto y 358 niñas de NSE bajo asistentes a tres escuelas públicas de la Región Metropolitana. Como se realizó en un estudio previo ${ }^{14}$, se seleccionó aleatoriamente un curso por nivel y se incluyó en la muestra a todas las niñas de los cursos seleccionados. El NSE al que pertenecían las niñas fue determinado con los antecedentes disponibles en los establecimientos. Los datos fueron analizados según grupos de edad: 8-9; 1011 y 12-13 años. En la Tabla 1 se muestra la distribución de las niñas según NSE y grupos de edad.

Tabla 1. D istribución de la muestra de escolares, según N SE y grupos de edad. Santiago, C hile 2004

\begin{tabular}{|lccc|}
\hline Grupos de Edad & Medio alto $(\mathrm{n}=204)$ & Bajo $(\mathrm{n}=358)$ & Total $(\mathrm{n}=562)$ \\
\hline $8-9$ & $71(35 \%)$ & $124(35 \%)$ & $195(35 \%)$ \\
$10-11$ & $52(25,5 \%)$ & $126(35 \%)$ & $178(31,7 \%)$ \\
$12-13$ & $81(39,7 \%)$ & $108(30,2 \%)$ & $189(33,6 \%)$ \\
\hline
\end{tabular}


Estado nutricional. Para evaluar el estado nutricional se comparó el índice de masa corporal (IMC) de cada niña según edad (en meses) con las tablas de $\mathrm{CDC} / \mathrm{NCHS}^{18}$, utilizando los puntos de corte actualmente aceptados por el Ministerio de Salud ${ }^{24}$ para evaluar el estado nutricional del menor de 18 años: bajo peso IMC percentil <10; peso normal IMC percentil $\geq 10-<85$; sobrepeso IMC percentil 85-94; obeso IMC percentil $\geq 95$. El peso y la estatura de las niñas fueron determinados por nutricionistas entrenadas, las que utilizaron una balanza Seca con tallímetro modelo 713, cuya escala presentaba una sensibilidad de $0,2 \mathrm{~kg}$ para el peso y $1 \mathrm{~mm}$ para la estatura.

Consumo de alimentos. Para estimar el consumo de alimentos, nutricionistas entrenadas aplicaron a cada niña una encuesta de frecuencia de consumo cuantificada (EFCC) validada en un estudio previo ${ }^{14}$. La aplicación de la encuesta tomó 25 a 30 min por niña. El consumo diario de algunos alimentos seleccionados (leche, yogur, verduras, frutas, pan; alimentos de alta densidad energética: papas fitas, chocolates, galletas dulces y saladas; bebidas con y sin azúcar) se calculó tomando como base el consumo semanal. Este tipo de encuesta ha sido recomendada como el mejor método para evaluar el consumo de alimentos que presentan una gran variabilidad día a día, porque no altera la conducta respecto a la ingesta de alimentos $^{25,26}$ y porque es apropiada para orientar los programas de educación en nutrición ${ }^{27}$. Para los fines de este estudio, los alimentos de alta densidad energética citados serán denominados snacks ${ }^{22,23}$. Considerando que el consumo de alimentos no tuvo una distribución normal, en la presentación de los resultados se utilizó la distribución percentilar.

Para obtener información confiable sobre la cantidad de alimentos consumidos por las niñas, las encuestadoras utilizaron un libro con imágenes de distintas porciones de alimentos ${ }^{28}$, cuya transformación a gramos fue previamente validada con escolares chilenos ${ }^{14}$. En el caso de los alimentos envasados, las encuestadoras consultaron por la marca del producto y obtuvieron la cantidad directamente del envase.

Hábitos de actividad física. Para estimar los hábitos de actividad física, se consultó a las niñas por el tiempo que veían televisión (TV) y la frecuencia con la que realizaban actividad física durante los días de colegio, al interior del mismo, en horario extracumicular o fuera del colegio, utilizando una encuesta validada en un estudio previo ${ }^{14}$. Cabe señalar que el colegio al que asistían las niñas de NSE medio alto tiene una completa infraestructura para las actividades deportivas, y ofrece a las alumnas actividades deportivas a su elección fuera de la jomada de clases, bajo la conducción de profesores especializados. El tiempo destinado a ver TV se consultó a través del número de programas vistos diariamente, como se ha hecho en estudios anteriores ${ }^{22,23}$.

Análisis estadístico. Para cada uno de los grupos de edad y NSE considerados en el estudio se calculó la distribución percentilar del consumo en gramos por día ( $\mathrm{g} /$ día) para los alimentos seleccionados. Las comparaciones del consumo por grupos de edad se realizaron con el test no paramétrico de KruskalWallis ${ }^{29}$. La comparación del consumo de alimentos de las niñas según NSE, se realizó con el test no paramétrico de Mann-Whitney. Para determinar diferencias entre el estado nutricional, horas de TV y actividad física con respecto a los grupos de edad y NSE, se utilizó el test de Chi-cuadrado o el test exacto de Fisher. El análisis estadístico de los datos se realizó en la Unidad de Estadística del INTA usando los programas Stata $8.2^{30}$ y SAS versión $8.2^{31}$.

\section{RESULTADOS}

Las Figuras 1a y 1b muestran la distribución del estado nutricional de las niñas según NSE y edad. En el grupo de 8-9 años, se observó una mayor prevalencia de sobrepeso (33,8\% vs $16,9 \%$ ) y menor de obesidad (8,5\% vs $18,6 \%)$ en las niñas de NSE medio alto con respecto a las de NSE bajo ( $p$ $=0,012$ ). En el grupo de 10-11 años no se observaron diferencias significativas ( $p=0,44$ ); el grupo de 12-13 años de NSE medio alto mostró una menor prevalencia de sobrepeso $(16,2 \%$ vs $25,9 \%)$ y obesidad (2,5\% vs $12 \%$ ), que el grupo de NSE bajo ( $p=0,008)$.

En la Tabla 2 se presenta la mediana y percentiles 25 y 75 de consumo diario de alimentos según NSE y edad. El consumo de lácteos fue significativamente mayor ( $p<0,0001$ ) en el grupo de NSE medio alto (mediana entre 450 y $493 \mathrm{ml} /$ día), sin diferencias por edad. En el NSE bajo, el consumo de lácteos disminuyó significativamente con la edad, desde 314 ml/día en las niñas de 8-9 años a $220 \mathrm{ml} /$ día en las 


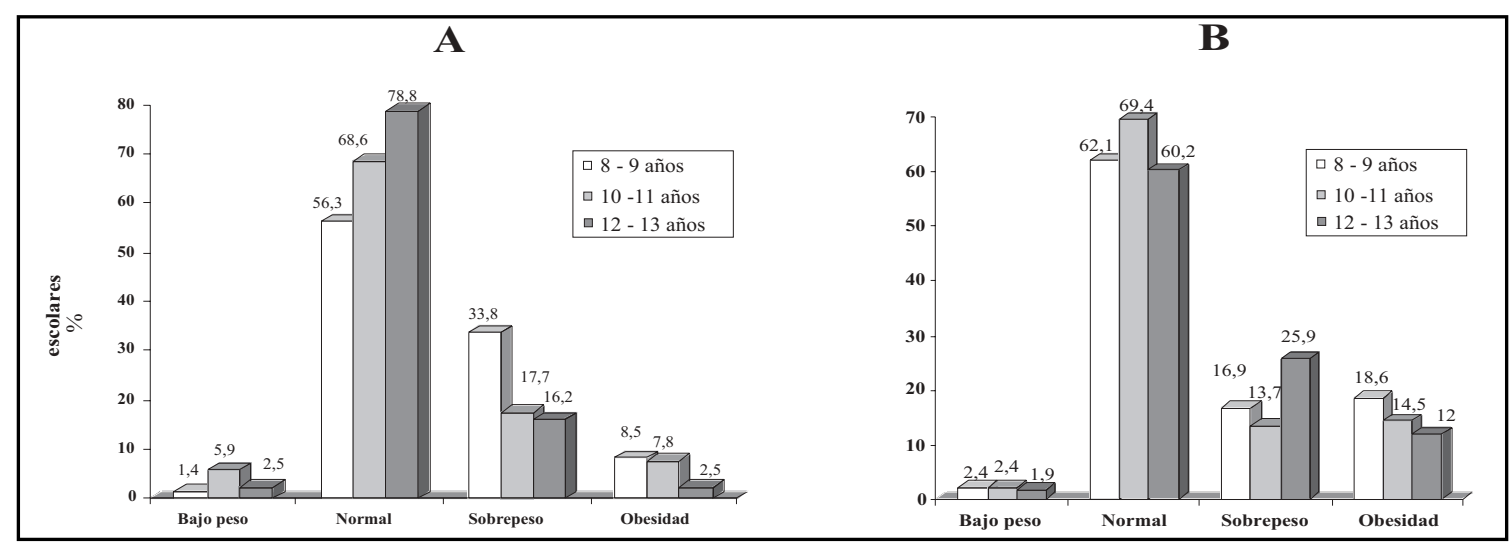

Figura 1. Estado nutricional de escolares mujeres según edad y su comparación por NSE ${ }^{1}$. Santiago, Chile 2004. A. NSE medio alto $(\mathrm{n}=202)$. B. NSE bajo $(\mathrm{n}=358)$.

${ }^{1}$ Test de Chi-cuadrado: $p=0,012$ (8-9 años). $p=0,44$ (10-11 años). $p=0,008$ (12-13 años).

Tabla 2. D istribución percentilar del consumo de alimentos seleccionados, según N SE y grupos de edad. Santiago, Chile 2004

\begin{tabular}{|c|c|c|c|c|c|c|c|}
\hline $\begin{array}{l}\text { Alimentos } \\
\text { g/día }\end{array}$ & Edad & $\begin{array}{c}\text { p25 } \\
\text { NSE } \\
\text { Medio } \\
\text { Alto }\end{array}$ & $\begin{array}{l}\text { NSE } \\
\text { Bajo }\end{array}$ & $\begin{array}{c}\mathrm{p} 50^{1} \\
\text { NSE } \\
\text { Medio } \\
\text { Alto }\end{array}$ & $\begin{array}{l}\text { NSE } \\
\text { Bajo }\end{array}$ & $\begin{array}{c}\text { p75 } \\
\text { NSE } \\
\text { Medio } \\
\text { Alto }\end{array}$ & $\begin{array}{l}\text { NSE } \\
\text { Bajo }\end{array}$ \\
\hline \multirow[t]{3}{*}{ Leche y yogurt } & 8-9 & 325 & 193 & 450 & $314^{* * *}$ & 575 & 450 \\
\hline & $10-11$ & 375 & 136 & 493 & $214^{* 2 k x}$ & 563 & 350 \\
\hline & $12-13$ & 304 & 143 & 474 & $220^{* 12 k}$ & 600 & 363 \\
\hline \multirow[t]{3}{*}{ Frutas y verduras } & 8-9 & 125 & 107 & 200 & $164^{*}$ & 250 & 229 \\
\hline & $10-11$ & 91 & 143 & 170 & 229 & 230 & 371 \\
\hline & $12-13$ & 107 & 143 & 196 & 229 & 290 & 414 \\
\hline \multirow[t]{3}{*}{ Pan } & $8-9$ & 29 & 167 & 71 & $217^{* 1 * k}$ & 100 & 300 \\
\hline & $10-11$ & 29 & 200 & 50 & $233^{*} * 1 \times$ & 107 & 308 \\
\hline & $12-13$ & 40 & 200 & 71 & $250^{* * * *}$ & 100 & 308 \\
\hline \multirow[t]{3}{*}{ Snacks dulces y salados } & $8-9$ & 36 & 48 & 73 & 68 & 109 & 101 \\
\hline & $10-11$ & 20 & 48 & 44 & $78^{* * * k}$ & 72 & 108 \\
\hline & $12-13$ & 32 & 52 & 51 & $85^{* * * 1}$ & 77 & 136 \\
\hline \multirow[t]{3}{*}{ Bebidas con azúcar } & $8-9$ & 0 & 114 & 200 & 207 & 500 & 286 \\
\hline & $10-11$ & 0 & 143 & 186 & 229 & 500 & 371 \\
\hline & $12-13$ & 0 & 143 & 171 & 229 ** & 500 & 414 \\
\hline
\end{tabular}

${ }^{1}$ Mann-Whitney Test *p <0,05; **p <0,001; **p $<0,0001$.

niñas de 12-13 años, respectivamente ( $p<0,0001)$. El consumo de verduras y frutas no mostró diferencias significativas (164 a $229 \mathrm{~g} /$ día), equivalentes a aproximadamente la mitad de lo recomendado ( $\geq 400 \mathrm{~g} /$ día $)^{31}$. El consumo de pan fue significativamente menor en las niñas de NSE medio alto en todos los grupos de edad (50 a 70 vs 217 a 250 g/día, respectivamente) ( $p<0,0001)$. El consumo de snacks fue semejante en las niñas de 8-9 años y significativamente menor en las niñas de NSE medio alto de 10-11 años (44 vs $78 \mathrm{~g} /$ día) y 12-13 años (51 vs $85 \mathrm{~g} /$ día, respectivamente) ( $p<0,0001$ ). El consumo de bebidas con azúcar fue significativamente menor en las niñas de 12-13 años de NSE medio alto (171 vs $229 \mathrm{ml} /$ día, respectivamente) ( $p=0,0024)$. Las niñas de NSE medio alto consumían además $180 \mathrm{ml} /$ día de 
bebidas sin azúcar, consumo observado por primera vez en nuestros estudios en escolares en diversas regiones del país.

Con respecto al tiempo destinado a mirar televisión (Figuras 2a y 2b), se observó que existe una asociación entre el NSE y las horas de TV en los tres grupos de edad ( $\mathrm{p}<0,05)$. En el grupo de 89 años, $17 \%$ de las niñas de NSE medio alto y $13 \%$ de las de NSE bajo veían más de tres horas de TV durante los días de colegio, en cambio en las niñas de 10-11 y $12-13$ años, 48 y $41 \%$ de las de NSE medio alto y 27 y $38 \%$ de las de NSE bajo veían 3 o más horas diarias de TV, respectivamente.

Al consultar por la frecuencia con la que realizaban actividad física durante los días de colegio, no se observaron diferencias significativas según NSE (Figuras 3a y 3b) ( $p>0,05)$. En ambos grupos, se observó que las niñas más activas eran las de 8-9 años (45\% realizaba actividad física 4 o más veces por semana), $\mathrm{y}$ las de 12-13 años las más inactivas (14,8\% y 19,2\% de las niñas de NSE medio alto y bajo nunca realizaban actividad física, respectivamente).

\section{DisCUSIÓN}

Al comparar el estado nutricional de las escolares según NSE, se observó una menor prevalencia de obesidad en las niñas de NSE medio alto en todos los grupos de edad, siendo más marcada en las niñas de 12-13 años. Al respecto, Burrows y cols ${ }^{32}$ han señalado que las menores proporciones de obesidad en el período de la pubertad podrían deberse a que el punto de corte del indicador IMC de la referencia $\mathrm{CDC} / \mathrm{NCHS}^{18}$ en la que se basó la norma chilena ${ }^{24}$, exige demasiado peso para ser clasificado como obeso, exigencia que es mayor para las niñas. Sin embargo, ambos grupos fueron evaluados con el mismo criterio, lo que no explicaría la gran diferencia observada. En estudios realizados en Estados Unidos de Norteamérica, se ha postulado la relación entre la prevalencia de obesidad y la existencia de factores socioeconómicos y culturales que afectan la autoimagen y el control del peso, los que a su vez podrían afectar la información sobre la ingesta de alimentos y la actividad física ${ }^{33-35}$.

$\mathrm{Al}$ analizar el consumo de lácteos, se observó que las niñas de NSE medio alto presentaron un consumo cercano a $80 \%$ de lo recomendado en las Guías Alimentarias chilenas ${ }^{36}$, semejante a lo observado en Estados Unidos de Norteaméri$\mathrm{ca}^{37,38}$, en cambio en las niñas de NSE bajo, el consumo alcanzó sólo a $50 \%$ de lo recomendado en las de 8-9 años, y a 37\% después de esa edad, similar a lo observado en otros estudios nacionales ${ }^{14,17,21}$.

En verduras y frutas, el consumo en ambos grupos fue semejante, $\mathrm{y}$ al igual que en estudios previos $^{14,19}$, alcanzó aproximadamente a $50 \%$ de lo recomendado ${ }^{36}$. Esta situación coincide con lo observado en otros países 37,39 y ha motivado la creación de programas específicos para promover un aumento en el consumo de estos alimentos ${ }^{40}$.

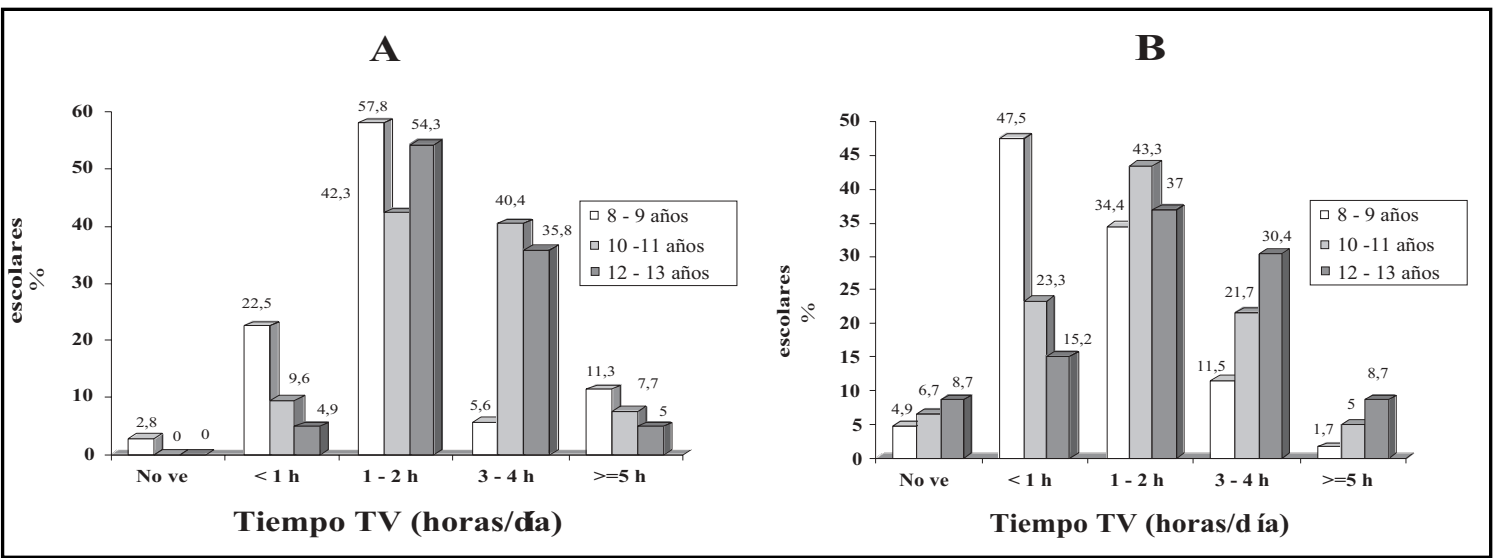

Figura 2. Tiempo que las niñas ven TV durante los días de colegio, según edad y NSE ${ }^{1}$. Santiago, Chile 2004. A. NSE medio alto $(\mathrm{n}=202)$. B. NSE bajo $(\mathrm{n}=358)$.

${ }^{1}$ Test de Chi-cuadrado: $p=0,002$ (8-9 años). $p=0,036$ (10-11 años). $p=0,008$ (12-13 años). 


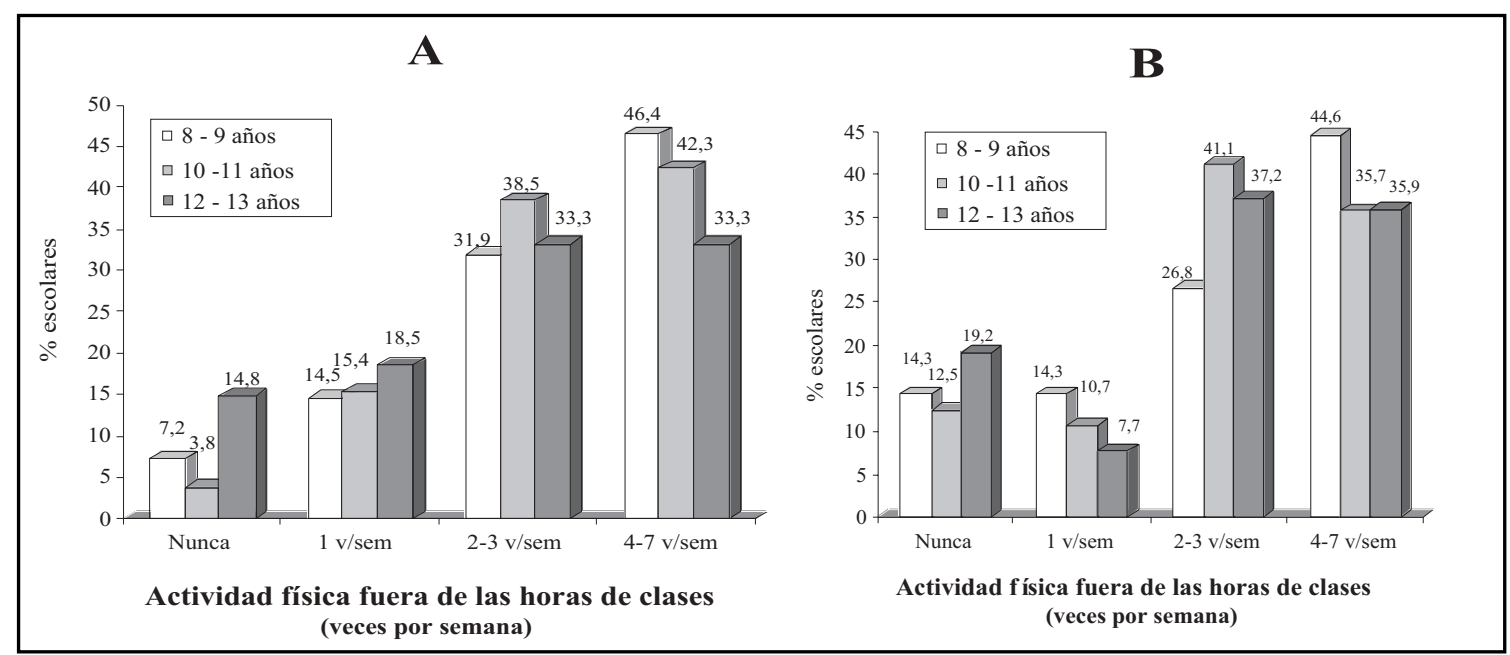

Figura 3. Frecuencia de actividad física en escolares mujeres durante los días de colegio, según edad y comparación por NSE ${ }^{1}$. Santiago, Chile 2004. A. NSE medio alto $(n=202)$. B. NSE bajo $(n=358)$.

${ }^{1}$ Test de Chi-cuadrado: $\mathrm{p}>0,05$ para todos los grupos de edad.

Llamó la atención el bajo consumo de pan en las niñas de NSE medio alto, significativamente menor que en las de NSE bajo. La explicación para el bajo consumo expresada por las niñas de NSE medio alto fue «mi mamá no quiere que coma más para que no engorde». Esta respuesta fue entregada también en forma espontánea para diversos snacks. Si bien el consumo promedio de éstos también fue significativamente inferior al encontrado en las niñas de NSE bajo, sigue representando un aporte importante a la ingesta energética total. El consumo de bebidas gaseosas fue semejante al encontrado en el grupo de menor NSE, excepto a los 12-13 años, cuyo consumo fue menor en las niñas de NSE medio alto. La influencia de las madres sobre la ingesta de alimentos y el control del peso en los niños es actualmente objeto de estudio y controversia, pero se ha postulado que podría estar relacionada con factores socioeconómicos y diferencias raciales, que cambian la percepción de la autoimagen y el menor o mayor rechazo al sobrepeso y la obesidad $34,35,40,41$.

La proporción de niñas que miraban 3 o más horas diarias de TV durante los días de colegio, fue muy superior en las mayores de 10 años que en las menores de esa edad y mayor en las NSE medio alto ( $48 \%$ y $41 \%$ en las niñas de NSE medio alto de $10-11$ y $12-13$ años versus $27 \%$ y $38 \%$ en las de NSE bajo, respectivamente). Esto representa un mayor número de horas frente al televisor que la observada en estudios anteriores ${ }^{14,22,23}$. Se ha señalado que los niños que pasan más de dos horas diarias frente al televisor son más sedentarios y tienen un mayor riesgo de obesidad ${ }^{43}$. En este contexto, la OMS recomienda a los gobiemos fomentar la actividad física y regular la publicidad de alimentos y bebidas, con el fin de crear un ambiente más favorable a la adquisición de hábitos alimentarios apropiados en los niños ${ }^{44}$.

$\mathrm{Si}$ bien la proporción de niñas que señaló no realizar actividad física fue mayor en las de NSE bajo, y que entre las que realizaban actividad física 4 o más veces por semana el porcentaje fue mayor en las de 8-9 años (45\%) que en las de 1213 años (35\%), las diferencias no fueron significativas. La falta de actividad física es considerada actualmente un factor de riesgo independiente de enfermedades cardiovasculares y las recomendaciones indican que el tiempo destinado a las actividades moderadas e intensas debería ser de al menos 60 min diarios en los niños ${ }^{3,45}$.

Los resultados de este estudio muestran que las niñas de ambos niveles NSE requieren intervenciones educativas orientadas a mejorar su alimentación y aumentar la actividad física para 
alcanzar un mejor estado nutricional. En el país existen propuestas validadas en educación en nutrición y actividad física ${ }^{15,46}$, que están disponibles para ser implementadas a nivel nacional. Sin embargo, se reconoce que las intervenciones

\section{REFERENCIAS}

1. WHO. Obesity, preventing and managing the global epidemia. Report of a WHO Consultation, June 3-5-1997. Geneva: World Health Organization; 1998.

2. LoBSTEIN T, BAUR L, UAUY R. Obesity in children and young people: a crisis in public health. Obes Rev 2004; 5 (Suppl 1): 4-85.

3. WHO. Diet, nutrition and the prevention of chronic diseases. Report of a Joint WHO/FAO Expert Consultation. Geneva: WHO; 2003 (WHO Serial Technical Report; 916).

4. Freedman D, Dietz W, SRinivasan S, Berenson G. The relation of overweight to cardiovascular risk factors among children and adolescents. The Bogalusa Heart Study. Pediatrics 1999; 103: 1175-82.

5. Must A, Strauss RS. Risks and consequences of childhood and adolescent obesity. Int J Obes Relat Metab Disord 1999; 23 (Suppl. 2): S2-S11.

6. Epstein LH, Coleman KJ, Myers MD. Exercise in treating obesity in children and adolescents. Med Sci Sports Exerc 1996; 28: 428-35.

7. Rippe JM, CRossiey S, Ringer R. Obesity as a chronic disease: modern medical and lifestyle management. J Am Diet Assoc 1998; 98 (Suppl. 2): S9-S15.

8. Dietz WH, GoRTMaker SL Do we fatten our children at the television set? Obesity and television viewing in children and adolescents. Pediatrics 1985; 75: 807-12.

9. Kiedgees R, Shelton M, Kiesges L. Effects of television on metabolic rate: potential implications for childhood obesity. Pediatrics 1993; 91: 281-6.

10. Robinson T, Hammer L, KiLen J, Kraemer HC, Wilson DN, Hayward C ET aL. Does the television viewing increase obesity and reduce physical activity? Cross-sectional and longitudinal analyses among adolescent girls. Pediatrics 1993; 91: 273-80.

11. Kotz K, Story M. Food advertisement during children's Saturday morning television programming: are they consistent with dietary recommendations? J Am Diet Assoc 1994; 94: 1296-300. educativas por sí solas no lograrán los cambios requeridos si los escolares no cuentan con ambientes apropiados y el apoyo necesario para llevar a la práctica las conductas saludables en el hogar, la escuela y la comunidad.

12. Young LR, NeStLe M. The contribution of expanding portion size to the US obesity epidemic. Am J Public Health 2002; 92: 246-9.

13. Kain J, Uauy R, Lera L, Taibo M, Espejo F, Albala C. Evolución del estado nutricional de escolares chilenos de 6 años (1987-2003). Rev Méd Chil 2005; 133: 1013-20.

14. Olivares S, Kain J, Lera L, Pizarro F, Vio F, Morón C. Nutritional status, food consumption and physical activity among Chilean school children: a descriptive study. Eur J Clin Nutr 2004; 58: 1278-85.

15. Kain J, Vio F, Leyton B, Cerda R, Olivares S, Uauy R ET AL. Estrategia de promoción de la salud en escolares de educación básica municipalizada de la comuna de Casablanca, Chile. Rev Chil Nutr 2005; 32: 126-32.

16. Kain J, Olivares S, Romo M, Leyton B, Vio F, Cerda $\mathrm{R}$ ET AL. Estado nutricional y resistencia aeróbica en escolares de educación básica: línea base para un proyecto de promoción de la salud. Rev Méd Chil 2004; 132: 1395-402.

17. Yáñez R, Oluvares S, Torres I, Guevara M, Díaz N. Consumo de alimentos en escolares chilenos. Su relación con las guías y la pirámide alimentaria. Rev Chil Nutr 2001; 28: 422-8.

18. CDC/NCHS. CDC growth charts: United States. http://www.cdc.gov/growthchart. Posted May 30, 2000.

19. Olivares S, Zacarías I, Lera L, Leyton B, Durán R, VIo F. Estado nutricional y consumo de alimentos seleccionados en escolares de la Región Metropolitana: línea base para un proyecto de promoción del consumo de pescado. Rev Chil Nutr 2005; 32: 102-8.

20. Kain J, Olivares S, Castimo M, Vio F. Validación y aplicación de instrumentos para evaluar intervenciones educativas en obesidad infantil. Rev Chil Pediatr 2001; 72: 308-18.

21. Atalah E, Urteaga C, Reboledo A, Delfín S, Ramos R. Patrones alimentarios y de actividad física en escolares de la región de Aysén. Rev Chil Pediatr 1998; 70: 483-90. 
22. Oluvares S, Albala C, García F, Jofré I. Publicidad televisiva y preferencias alimentarias en escolares de la Región Metropolitana. Rev Méd Chil 1999; 127: 791-9.

23. Olivares S, Yáñez R, Díaz N. Publicidad de alimentos y conductas alimentarias en escolares de $5^{0} \mathrm{a}$ 8o básico. Rev Chil Nutr 2003; 30: 36-42.

24. Ministerio de SAlud, Unidad de Nutrición, Consejo Asesor de Nutrición, Grupo de ExperTos. Norma técnica de evaluación nutricional del niño de 6 a 18 años. Rev Chil Nutr 2004; 31: 128-37.

25. Thompson F, Byers T. Dietary assessment resource manual. J Nutr 1994; 124 (11 Suppl): 2245S-2317S.

26. WiLET W. Future directions in the development of food-frequency questionnaires. Am J Clin Nutr 1994; 59 (Suppl): 171S-174S.

27. Van Staveren WA, Burema J. Validity of dietary survey methods: a critical approach. In: Schürch $B$, editor. Evaluation of nutrition education in third world communities. A Nestlé Foundation Workshop. Switzerland: Hans Huber Publishers; 1982 (Nestlé Foundation Publication Series, vol. 3: 69-79)

28. VÁsQuez M, Witriw A. Modelos visuales de alimentos. Tablas de relación peso/volumen. Buenos Aires: Universidad de Buenos Aires; 1997.

29. HeRnándeZ R, Fernández C, BAptista P. Metodología de la investigación. $2^{\underline{a}}$ ed. México: Mc Graw-Hill Interamericana Editores; 2001.

30. STATA. Stata 8.2. Stata Corporation. College Station, USA; 2004.

31. SAS. SAS Institute Inc. (2002), SAS/ STAT Software: user's guide, Release 8.2. Cary, NC: SAS Institute, Inc.

32. Burrows R, DíAz N, Muzzo S. Variaciones del índice de masa corporal de acuerdo al grado de desarrollo puberal alcanzado. Rev Méd Chil 2004; 132:1363-8.

33. Sams J, Buono J, Roby J, Micale F, Nelson J. Sevenday recall and other physical activity self-reports in children and adolescents. Med Sci Sports Exerc 1993; 25: 99-108.

34. Fisher J, Johnson R, Lindquist C, Birch L, Goran M. Influence of body composition on the accuracy of reported energy intake in children. Obes Res 2000; 8: 597-603.

35. Kiesges L, Baranowski T, Beech B, Culen K, Murray D, Rochon J et AL. Social desirability bias in self- reported dietary, physical activity and weight concerns measures in 8-10 year old AfricanAmerican girls: results from the Girls health Enrichment Multisite Studies (GEMS). Prev Med 2004; 38: S78-S87.

36. Ministerio de Salud/INTA/Vida Chile. Guía para una vida saludable. Santiago; Ministerio de Salud; 2005. Norma 76.

37. Kennedy E, Ohis J, Carlson S, Fleming K. The healthy eating index: Design and applications. J Am Diet Assoc 1995; 95: 1103-8.

38. United States Department of Agriculture, United States Department of Health and Human Services. Dietary guidelines for Americans 2000. Washington DC: USDA 2000. http:// www.cnpp.usda.gov/Pubs/DG2000.

39. Aranceta J, Pérez-Rodrigo C, Serra-Majem L. Sociodemographic and lifestyle determinants of food patterns in Spanish children and adolescents: the enKid study. Eur J Clin Nutr 2003; 57 (Suppl 1): S40-S44.

40. US Department of Health and Human Services, National Institutes of Health, National Cancer Institute. The $5 \mathrm{~A}$ Day for Better Health program. Washington DC: US Department of Health and Human Services 2004. http://www.5aday.gov/ about/index.html.

41. BIRCH LL, FISHER JO. Mothers' child feeding practices influence daughters's eating and weight. Am J Clin Nutr 2000; 71: 1054-61.

42. Robinson TN, Kierman M, Matheson DM, Haydel KF, Is parental control over children's eating associated with childhood obesity? Results from a population-based sample. Obes Res 2001; 9: 306-12.

43. Andersen RE, Crespo CJ, Bartlett SJ, Cheskin LJ, PRATT M. Relationship on physical activity and television watching with body weight and level of fatness among children. J Am Med Assoc 1998; 279: 938-42.

44. World Health Organization. Marketing food to children: the Global Regulatory Environment. Geneva: WHO 2004.

45. Biair S, LaMonte M, Nichaman M. The evolution of physical activity recommendations: how much is enough? Am J Clin Nutr 2004; 79 (Suppl): 3S-20S.

46. Olvares S, Zacarías I, Andrade M, Kain J, Lera L, Vio $F$ ET AL. Nutrition education in Chilean primary schools. Food Nutr Bull 2005; 26: S179-S185. 